\title{
Recent advances in theoretical models of respiratory mechanics
}

\author{
Bo Huo • Rui-Rong Fu
}

Received: 29 September 2011 / Revised: 21 December 2011 / Accepted: 5 January 2012

(C)The Chinese Society of Theoretical and Applied Mechanics and Springer-Verlag Berlin Heidelberg 2012

\begin{abstract}
As an important branch of biomedical engineering, respiratory mechanics helps to understand the physiology of the respiratory system and provides fundamental data for developing such clinical technologies as ventilators. To solve different clinical problems, researchers have developed numerous models at various scales that describe biological and mechanical properties of the respiratory system. During the past decade, benefiting from the continuous accumulation of clinical data and the dramatic progress of biomedical technologies (e.g. biomedical imaging), the theoretical modeling of respiratory mechanics has made remarkable progress regarding the macroscopic properties of the respiratory process, complexities of the respiratory system, gas exchange within the lungs, and the coupling interaction between lung and heart. The present paper reviews the advances in the above fields and proposes potential future projects.
\end{abstract}

Keywords Respiratory mechanics $\cdot$ Biomechanics · Gas exchange

\section{Introduction}

Respiratory mechanics simulates and predicts the dynamics process of a respiratory system. The investigations may help understand the pathophysiology of some respiratory dis-

The project was supported by the National High Technology Research and Development Program of China (2009AA02Z407) and the National Natural Science Foundation of China (30970707).

\footnotetext{
B. Huo (凶) · R.-R. Fu

Key Laboratory of Microgravity \& Center for Biomechanics and Bioengineering,

Institute of Mechanics, Chinese Academy of Sciences, 100190 Beijing, China

e-mail: huobo@imech.ac.cn
}

eases, such as acute respiratory distress syndrome (ARDS) and chronic obstructive pulmonary disease (COPD). Furthermore, respiratory mechanics may provide theoretical support for designing novel medical devices such as respiratory ventilators. Considering the complexity of the human respiratory system, theoretical modeling is very critical in the study of respiratory mechanics. Usually, the adopted model determines what is observable in an experiment and how a numerical simulation is performed. A successful model has to extract fewer but more significant parameters, preferably through non-invasive methods, and is able to provide adequately accurate descriptions of the respiratory process. More importantly, the model should be easy to use for clinicians. In the past decade, biomedical imaging technologies have made dramatical progress and a large number of advancements have been achieved in mechanics, physiology, and lung pathology. Benefiting from these advancements, respiratory mechanics also achieved remarkable progress regarding lung mechanobiology [1], disease-related mechanics [2], air-liquid two-phase flow in airway [3], image-based modeling of lung structure and function [4], and so on. The present paper reviews recent advances in the theoretical models involving macroscopic modeling, complexity of the respiratory system, gas exchange, and heart-lung coupling which are shown in Fig. 1a. The basic concepts and equations of some typical models are presented and their respective advantages and limitations are also provided, as well as the cases in which they are properly suitable adopted. Finally, several potential future projects are suggested.

\section{Macroscopic models}

The primary function of the respiratory system is to supply oxygen and carry away carbon dioxide from the blood. When only a quantitative evaluation of the efficiency of the respiratory system is required, it is usually sufficient just to determine the oxygen consumption and carbon dioxide production during a time interval. In this case, mobile human 
bodies or animals are in a chamber and the contents of gases at the inlet or the outlet are monitored (Fig. 1b). The mass balance of nitrogen requires that the measured sample does not store or release nitrogen. Therefore, the oxygen consumption rate can be evaluated using the following equation [5]

$\dot{V}_{\mathrm{O}_{2}}=\dot{V}_{\mathrm{I}} \cdot\left[F_{\mathrm{IO}_{2}}-\frac{F_{\mathrm{EO}_{2}}\left(1-F_{\mathrm{IO}_{2}}-F_{\mathrm{ICO}_{2}}\right)}{1-F_{\mathrm{EO}_{2}}-F_{\mathrm{ECO}_{2}}}\right]$,

where $\dot{V}_{\mathrm{I}}$ is the inlet flux, and $F_{\mathrm{IO}_{2}}, F_{\mathrm{ICO}_{2}}, F_{\mathrm{EO}_{2}}$, and $F_{\mathrm{ECO}_{2}}$ are the oxygen and carbon dioxide content at the inlet and outlet, respectively. This type of model does not focus on the detailed process of a respiratory cycle, but instead regards the whole sample as a black box and evaluates the system parameters using the variables at its interface with the external environment. One of the advantages of these models is that they are capable of obtaining the energy loss of freely mobile organisms. Recently, Halsey et al. [6] developed a model by combining overall dynamic body acceleration (ODBA) and comparing the oxygen consumptions of several animals at different accelerations to assess indirectly the efficiency of the respiratory process and lung function.

Clinicians usually analyze pressure-volume $(P-V)$ curves to estimate the elastic properties of the respiratory system, to consider the variations of physiologic or biochemical factors, e.g., alveolar surfactants [7] and shunts [8]. In the current study, the pressure refers to the difference between airway pressure $P_{\mathrm{AW}}$ and pleural pressure $P_{\mathrm{Pl}}$. The relations between this pressure difference and the gas volume $V$ inspired into the airway can be written as follows [9]

$P_{\mathrm{AW}}=P_{\mathrm{Pl}}+\gamma \dot{V}+V / C$,

where $\gamma$ is the airway resistance, and $C$ the lung compliance. This equation indicates that the pressure at the airway inlet overcomes the pleural pressure, airway resistance, and alveolar elasticity. Considering the airway resistance is only $1 \%$ of the pressure required to inflate the pulmonary alveoli, the second term on the right side of Eq. (2) is usually ignored.

The $P-V$ relationship in a normal lung is linear within a range of tidal volume but nonlinear in a diseased lung [9]. For the nonlinear $P-V$ curves, the linear or multiple linear regression underestimates lung compliance during the early inspiratory process. Therefore, a sigmoidal function is usually adopted such as [7]

$V=a+\frac{b}{1+\mathrm{e}^{-\left(P_{M}-c\right) / d}}$,

where the fitting parameter $a$ denotes the lower asymptote volume, $b$ is the total change in volume between the lower and the upper asymptotes, $c$ is the pressure at the inflection point of the sigmoidal curve, and $d$ is the parameter proportional to the pressure range within which most of volume changes take place (Fig. 1c). This model can fit very well the process of lung expansion and retraction, and it is capable of diagnosing lung diseases, such as ARDS. Furthermore, setting an appropriate positive end expiratory pressure (PEEP) is helpful when using ventilators [10]. Recently, Schumann et al. [11] used a gliding-SLICE method to fit $P-V$ curves, which is able to evaluate the local continuous nonlinear compliance of the lungs.

Both the airways and lungs are viscoelastic materials, thus, Kent et al. recently developed a viscoelastic mechanical model [12], in which the change of airway pressure depends on the gas velocity as follows

$P_{\mathrm{AW}}(t)=\int_{-\infty}^{t} G(t-\tau) \frac{\partial P_{\mathrm{S}}(V)}{\partial V} \frac{\partial V(\tau)}{\partial \tau} \mathrm{d} \tau$,

where $P(t)$ is the airway pressure at time $t, P_{\mathrm{S}}(t)$ describes the pressure response to a step increase of air volume, and $G(t)$ is a monotonically decreasing reduced relaxation function. Compared with the experimental results in swine, this model successfully mimics the pressure variations during the respiratory process.

These macroscopic models are simple, with limited parameters, easy to understand, and convenient to use. They can qualitatively evaluate respiratory function; however, it is difficult to predict special phenomena induced by the complexity of the respiratory system. Moreover, they are unable to describe the gas exchange at the alveoli and the alterations of lung function related to lung diseases.

\section{Models considering the complex structure of a respira- tory system}

As gas passes the tree-like bifurcated airway (Fig. 1d) before arriving at the alveoli, the fluid dynamics of gas in this complex structure is very complicated. The most usually used method for solving this problem is the lumped parameter model, which simplifies the airway as a series of compartments, then extracts their mechanical properties [2], connects them by referring to the geometric and mechanical boundary conditions, and finally determines the lumped parameters of each compartment. These lumped parameters include resistance, inertia (that is, the pressure gradient that alters flux, which depends on fluid density, tube length, and sectional area), compliance, pressure, and volume, among others (Fig. 1e). They can be analogous to the parameters of an electric circuit, such as air resistance with electric resistance, inertia with electric induction, compliance with electric capacity, pressure with voltage, and volume with electric current.

Various methods have been used to divide the compartments. Barbini et al. [13] divided the trachea and bronchi as three continuous segments, and simulated the flow ratevolume curves of normal subjects and patients with COPD to understand the mechanism of expiratory flow limitation (EFL) under mechanical ventilation. Antonaglia et al. considered the left and right lobes as two compartments with distinct elastic and viscoelastic material properties (Fig. 1e), through which they studied the gas distribution in the two compartments during constant-flow controlled volume in- 
flation (VCV) and inspiratory constant pressure inflation (PCV). They found that PCV results in slightly higher airtrapping than VCV, which shows that the viscoelastic properties of the lungs influence the gas distribution in different compartments [14].

Based on the multi-scale geometric characteristics of the airway, Nacci et al. [15] suggested an anatomically consistent model in the time domain to describe lumped parameters, such as the compliance of the airway tube and the resistance of laminar flow within. The authors considered the heterogeneous constraints on the small airways and used a second-order viscoelastic model at alveolar level to represent the stress adaptation behavior of lung tissue. This method facilitates studies on the effect of homogeneous and inhomogeneous constrictive pathology, as well as different ventilation strategies on the distribution of pressure and air flow in the alveoli. Polak and Mroczka [16] developed a model to calculate the pressure drop, then analyzed the local nonlinear effects and the dynamic properties of the airway. Among their considerations were the morphology of the airway, the dynamic behavior of the lungs and chest wall, the nonlinearity related to the turbulence of the air flow, and the collapse and the time-variable mechanical properties of the airway.

In the past decade, dramatic development of bioimaging technologies made it possible to obtain true structure of the respiratory system. For example, Lo et al. [17] developed a novel technique of image processing and analysis through which the fine structure of the airway tree can be visualized from low-dose CT images. Comerford et al. [18] established a three-dimensional model of the lungs to study the airflow in patient-specific human lungs and found that the flow depends mainly on the lower generation of the airway tree rather than on the peripheral vessels. This type of elaborate model of lung airways is suitable for the numerical analysis of fluid dynamics. For example, Wilson et al. [19] conducted fluid dynamics simulations to obtain the corresponding dynamic variables at the two ends of the upper airway, which was further connected with other models to predict the function of the entire respiratory system. When predicting the gas distribution in the lungs, the mechanical computation basing on a true structure is obviously more accurate than the traditional hypothesis of constant velocity or pressure boundary conditions [20].

The mechanical properties of the pulmonary alveoli, e.g., high shear strain, fatigue failure during opening and collapse, and the overdistension of the alveolar membrane, play key roles in respiratory function. When considering the opening and collapse of pulmonary alveoli, $P-V$ curves of the respiratory system reveal its oscillation properties [21]. Smith et al. [22] assumed that the alveoli in overdistension and collapse are not involved in gas exchange, i.e., they are regarded as part of the dead space. They then obtained important physiological parameters such as maximum alveolar volume and alveolar compliance by fitting the $P-V$ curve of an ARDS patient. Inappropriate mechanical ventilation may cause lung injury. One way of solving this problem is to increase the inspiratory pressure to inflate the alveoli in collapse, which retains a PEEP that keeps alveoli open. However, the issue of adopting this technology as a routine clinical treatment is still under debate. It is very important to study the shear stress and dynamics around pulmonary alveoli and local instability of an alveolus. One recent study considered a structure with two connecting alveoli with equal pressure, wherein the $P-V$ curves of each alveolus are the same [23]. Stability analysis of the structure showed that alveoli with higher membrane tension collapse before the others.

The above models all took into account the complexity of the respiratory system. These efforts will provide more explanations on the effects of disease-induced structural variations in respiratory system functions. Using an engineering point of view to simplify a respiratory system as compartments simplifies the models, but there are still some problems. For instance, most of the current models do not have specific physiologic and clinical significance and the corresponding parameters are also difficult to measure, which makes the models inconvenient for application to specific diseases. Numerical simulations based true structures from CT images provide more accurate fluid dynamics properties of the air flow in the airways but they are limited by the computation capacity and the resolution of CT images. Analysis of alveolar stability may effectively contribute to the key problem of alveolar opening or collapse, but no studies concerning the relationship between the stability of alveoli and the gas exchange across alveolar membranes have been conducted.

\section{Models involving gas exchange}

Classical studies concerning gas exchange across alveolar membranes have been performed, but some recent researches have shed new lights. For example, one important parameter for assessing respiratory function is the oxygen content in pulmonary alveoli or oxygen partial pressure $P_{\mathrm{AO}_{2}}$. In 1946, Fenn et al. [24] established a widely used equation for $P_{\mathrm{AO}_{2}}$

$P_{\mathrm{AO}_{2}}=P_{\mathrm{IO}_{2}}+\frac{P_{\mathrm{ACO}_{2}}}{R}\left[F_{\mathrm{IO}_{2}}(1-R)-1\right]$,

where $P_{\mathrm{ACO}_{2}}$ is the partial pressure of carbon dioxide, $F_{\mathrm{IO}_{2}}$ is the oxygen content of the inhaled gas, and $R$ is the respiratory quotient, i.e., the volumetric ratio of the carbon dioxide and oxygen exchanged between the blood and alveoli. The difficulty of measuring $P_{\mathrm{ACO}}$ limits the application of this model. Aboabet al. [25] recently combined Eq. (5) with equations that describe the oxygen content in the blood or the oxyhemoglobin dissociation curve, and then established a correlation between $P_{\mathrm{AO}_{2}}$ and blood oxygen content. Some studies $[26,27]$ directly predicted the carbon dioxide content in the blood based on the airway pressure obtained through 
an empirical formula.

The alveolar diffusion parameter is also an important index that reflects the gas exchange ability in the lungs. The overall oxygen diffusion resistance $1 / D_{\mathrm{L}}$ depends on the resistances offered by the alveolar membrane, interstitial tissue, blood vessel wall, and plasma, as well as the reaction rate of oxygen with hemoglobin in erythrocytes (Fig. 1f). In 1957, Roughton and Forsters suggested a lumped-parameter model as follows [28]

$\frac{1}{D_{\mathrm{L}}}=\frac{1}{D_{\mathrm{Am}}}+\frac{1}{\theta V_{\mathrm{Pc}}}$,

where $D_{\mathrm{Am}}$ denotes the diffusion capacity of the gas-blood barrier, $\theta$ is the reaction rate of oxygen with hemoglobin, and $V_{\mathrm{Pc}}$ is the volume of capillary blood. Recently, Patel [29] found that the membrane diffusion capacity is largely independent of $P_{\mathrm{AO}_{2}}$ and the erythrocyte diffusion capacity $\left(\theta V_{\mathrm{Pc}}\right)$ increased steeply with decreasing $P_{\mathrm{AO}_{2}}$.

The ventilation/perfusion ratio is usually used to assess the gas exchange properties through pulmonary alveoli. Ventilation means the flow rate of gas arriving at the alveoli $(\mathrm{g} / \mathrm{min})$, and perfusion refers to the flow rate of blood around the alveoli $(\mathrm{L} / \mathrm{min})$. The ventilation/perfusion ratio has the same unit as concentration. The overall ventilation/perfusion ratio in a normal lung is regarded as 0.8 , which is called the ventilation-perfusion balance or matching. However, this ratio is inhomogeneous among different lung locations and is usually regarded as dependent on gravity and the position of a lung relative to the heart [30]. In 1917, the ventilationperfusion mismatch was first reported to lead to varying gas exchange efficiencies [31]. After that discovery, numerous studies demonstrated that this mismatch is the primary reason for hypoxemia and hypercapnia. The multiple inert gas elimination technique (MIGET) is the gold standard for characterizing the lung ventilation-perfusion distribution. It is performed by measuring the pulmonary exchange of a set of six different inert gases dissolved together in saline (or dextrose) and infused intravenously [32]. Recently, Anderson and Hlastala improved the composition of the inert gases, which reduced the gas exchange in the airway [33].

A number of models with various complexities have been developed to describe the gas exchange within the lungs. Such models include a model that compares the difference in carbon dioxide partial pressures in pulmonary artery and end-tidal carbon dioxide content [34], a mathematical model of gas transport in pulmonary blood vessels [35], a hierarchy of models for human lungs [36], a model combined with MIGET for studying gas exchange in an injured lung [37], a computational model that analyzes the effect of venous admixture on pulmonary dead space and carbon dioxide exchange in an ARDS patients [38], and others. Recently, Wilson et al. [19] proposed a multi-technique approach that includes a compartmental model for a ventilator, a three-dimensional fluid dynamics computation for the flow field in the upper airway, a Windkessel style balloon model for the mechanical lung properties, the bifurcating tree models for the conducting airways and the pulmonary circulation, and a non-linear algebraic model for the chemical reaction during gas exchange. This model predicts arterial blood gas and airflow parameters consistent with the measured values.

Although the above models are capable of predicting the distribution of gas ventilation and blood perfusion and indirectly evaluates the efficiency of gas exchange, no proposed model has been able to correlate directly the blood gas content during mechanical ventilation and the mechanics of the respiratory system. This is also the main reason why gas exchange is usually ignored in the control program of current respirators.

\section{Coupling models of cardiopulmonary system}

The cardiovascular and pulmonary systems usually interact mechanically (Fig. 1a). For example, the increased pleural pressure during expiration compresses the heart chamber, the pulmonary vein and artery, and systemic vein, which further influences blood output to the periphery and blood transfer into lung vessels. Conversely, during inspiration, the decrease in pleural pressure leads to the expansion of the right ventricle, right atrium, and the superior and inferior vena cavae, inside which the decreased blood pressure improves the input blood volume. Considering the common wall between the right and left ventricles, the expansion of the right portion of the heart compresses the left portion of the heart, which further decreases the blood volume in the left side of the heart. In addition, the right ventricle is connected to the upstream flow of the pulmonary artery. Thus, the beat frequency and strength of the right heart ventricle directly regulate the flow rate of pulmonary blood. The contraction of the heart also suppresses the diffusion of gases from the pulmonary alveoli into the blood. In contrast, the expansion of the heart promotes the flux of gases into the blood. The coupled cardiopulmonary interaction is therefore important for accurately evaluating the function of the respiratory system, especially for patients with cardiovascular diseases.

Recently, researchers began establishing respiratory mechanics models that considers the heart-lung interaction. Qiu and Bai [39] proposed a model that includes five modules to mimic the dynamics of blood circulation and gas exchange, i.e. the gases transportation, exchange, and storage module, the multi-element nonlinear model of blood circulation module, an alveolar ventilation controller, a cardiac output controller, and a breathing frequency controller. They investigated the interaction of blood dynamics and gas exchange using the proposed model. Other models adopted pulmonary alveolar pressure, pleural pressure, and abdominal pressure as the output of the respiratory process and regarded them as the input parameters of their cardiovascular model. And the coupled interaction between the lungs and 
b
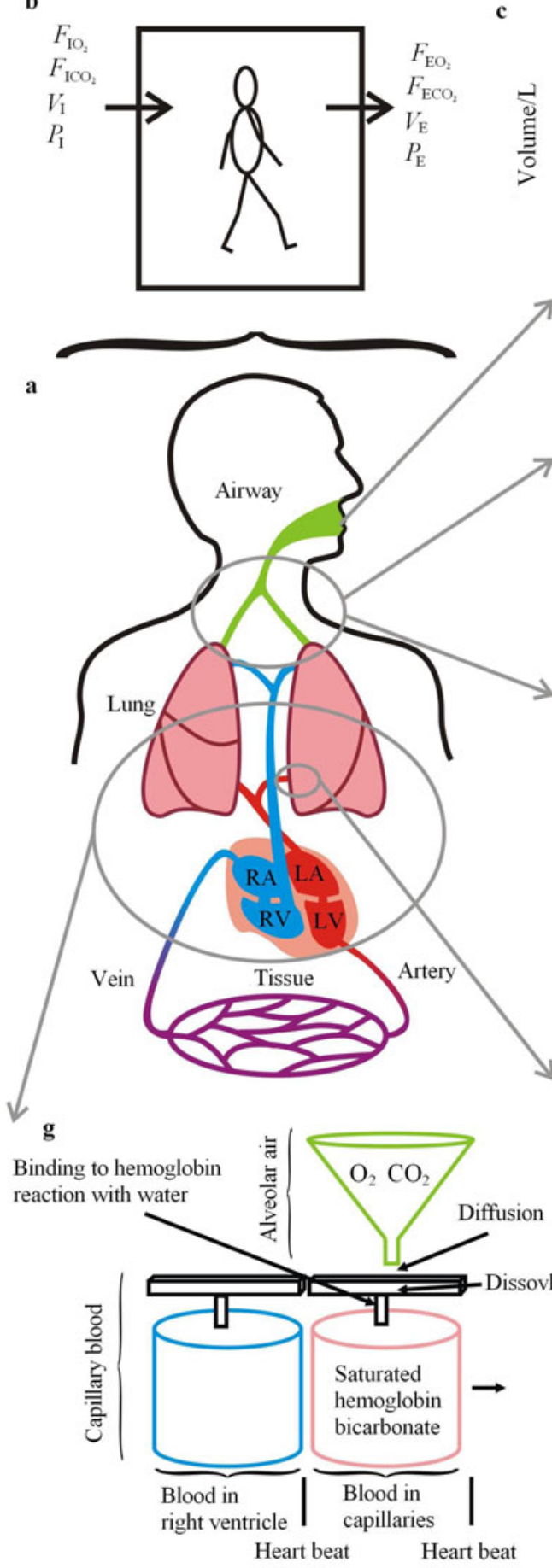

c

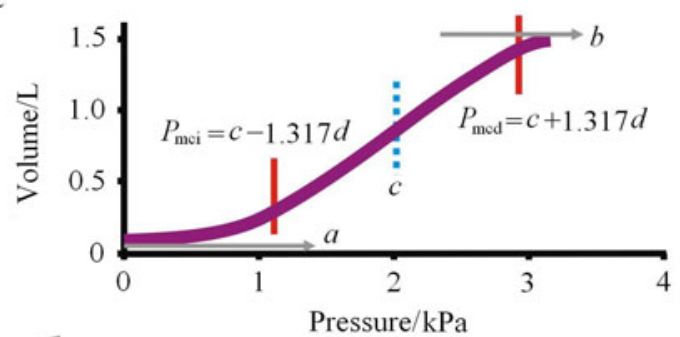

d

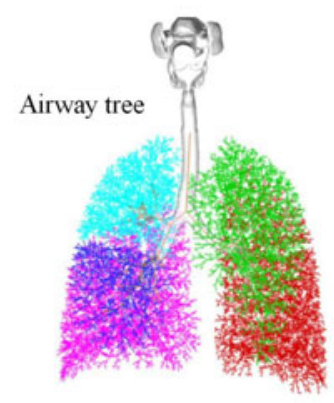

e

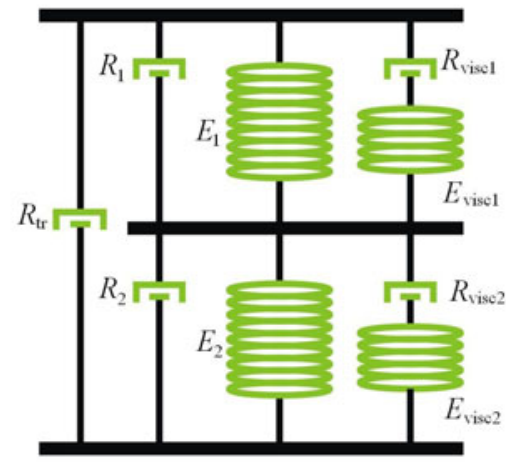

f

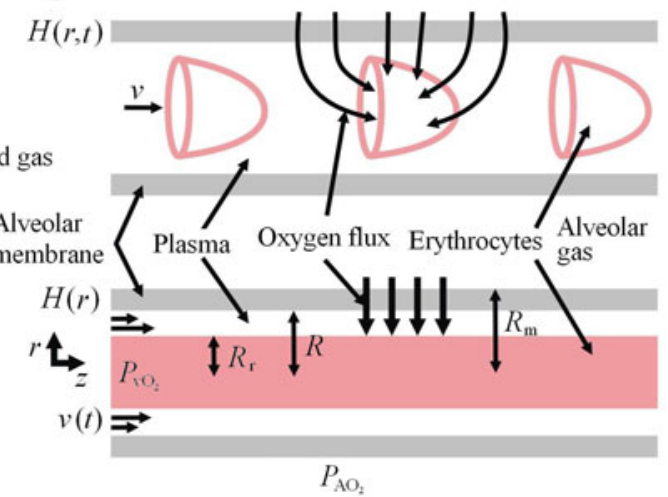

Fig. 1 Schematic drawing of typical respiratory mechanics models. a The respiratory system and blood circulation system, in which the arrows and circles show the position concerned with the corresponding model (RA, right atrium; RV, right ventricle; LA, left atrium; LV, left ventricle); b A macroscopic model that merely considers the oxygen consumption and carbon dioxide production of a mobile human body in a chamber; c A typical inflation $P-V$ curve in a patient with ARDS, including the parameters in a sigmoidal model [10]; d A generated 1D centerline airway tree containing the 3D CT-resolved upper airway, central airway tree, and central airway skeleton [20]; e A two-compartment lumped parameter model, in which the spring and dashpot represent the static elasticity and the resistance, and a module comprising a dashpot and a spring reflect the tissue viscoelasticity [14]; f A model showing the process of oxygen uptake of blood flowing through the pulmonary capillaries [29]; $\mathbf{g}$ A lung-heart coupling model that includes gas exchange between alveolar gas and blood, as well as blood gas transport [40] 
the heart was thus investigated [41, 42]. One recent study combined a ventilatory neuromuscular model and a cardiovascular model [43]. The authors used an oscillator to simulate the activities of the respiratory system and rib cage mechanics, inputting the change in pleural pressure produced by the respiratory process into the cardiovascular circulation, and inputting the blood volume in the pulmonary artery and vein produced by the heart output into a gas-blood exchange model. This model gave good predictions compared with experimental results. Ben-Tal assumed the right ventricle, pulmonary capillaries, and alveoli as compartments, and then modeled the process in which the heart rate regulated the cardiac output and the oxygen content in the pulmonary capillary. This model was also capable of analyzing the synchronized interaction between ventilation rate and heart rate (Fig. 1g) [36]. Ben-Tal and Smith [44] further improved their model by adding a neural feedback process, with which they considered the regulation action of neural rhythm on the respiratory muscles and subsequently investigated the effects of two delays caused by neural feedback and blood circulation on the respiratory system.

Investigating the interaction between the respiratory system and other biological systems is an exciting advancement in respiratory mechanics. This may indicate a novel therapeutic approach to lung disease, such as artificially controlling the rhythm of the neural system to influence the dynamic behavior of the respiratory system and regulating the gas exchange in the circulatory system. However, obtaining applicable models is still a long way because of insufficient experimental data.

\section{Future perspective}

The current review of recent advances indicates several trends in respiratory mechanics, such as improvement of accuracy in the evaluation of respiratory function, inclusion of the parameters that characterize the diseases and microstructures into the complex respiratory model, measurement and prediction of the gas exchange properties of the pulmonary alveoli, and establishment of the coupled interaction of the respiratory system with other physiologic systems, especially the circulatory system and the nervous system.

One of the barriers to the construction of a more accurate model is the limited measurement accuracy of clinical technologies. For example, a feasible and acceptable suitable technology for measuring the gas content in the pulmonary blood vessels should have enough time resolution to describe the variation trends of the parameters in a respiratory cycle. More importantly, it is critical to develop non-invasive techniques as routine clinical methods. One potential method is the measurement of gas content in peripheral blood and the prediction of the gas content in pulmonary blood before or after gas exchange. A good respiratory ventilator is usually intellectualized and appropriate for any specific individual patient, thus, the respiratory model controlling this machine should include more real-time physiologic parameters, such as heart rate, blood oxygen saturation, and end expiratory pressure. In addition, the achievements in the mechanobiology of molecules, cells, and tissues of the respiratory system should be employed in a respiratory mechanics model capable of correlating a disease with the mechanical properties of the respiratory system.

When these potential projects have been performed, we will be able to determine the general mechanical laws of the respiratory system, as well as predict and evaluate the function of individual respiratory systems based on the corresponding physiologic situation, ultimately providing better services for the welfare of human health.

\section{References}

1 Tschumperlin, D.J., Boudreault, F., Liu, F.: Recent advances and new opportunities in lung mechanobiology. J. Biomech. 43(1), 99-107 (2010)

2 Huang, R., Zhao, X., Rong, Q.: The advance of biomechanical studies of obstructive sleep apnea syndrome. Advances in Mechanics 40(3), 298-308 (2010)

3 Tavana, H., Kuo, C.H., Lee, Q.Y., et al.: Dynamics of liquid plugs of buffer and surfactant solutions in a micro-engineered pulmonary airway model. Langmuir 26(5), 3744-3752 (2010)

4 Tawhai, M.H., Lin, C.L.: Image-based modeling of lung structure and function. J. Magn. Reson. Imaging 32(6), 1421-1431 (2010)

5 Withers, P.C.: Design, calibration and calculation for flowthrough respirometry systems. Aust. J. Zool. 49(4), 445-461 (2001)

6 Halsey, L.G., Shepard, E.L.C., Quintana, F., et al.: The relationship between oxygen consumption and body acceleration in a range of species. Comp. Biochem. Phys. A 152(2), 197202 (2009)

7 Venegas, J.G., Harris, R.S., Simon, B.A.: A comprehensive equation for the pulmonary pressure-volume curve. J. Appl. Physiol. 84(1), 389-395 (1998)

8 Caramez, M.P., Kacmarek, R.M., Helmy, M., et al.: A comparison of methods to identify open-lung PEEP. Intensive Care Med. 35(4), 740-747 (2009)

9 Nikischin, W., Gerhardt, T., Everett, R., et al.: A new method to analyze lung compliance when pressure-volume relationship is nonlinear. Am. J. Respir. Crit. Care Med. 158(4), 1052-1060 (1998)

10 Pereira, C., Bohe, J., Rosselli, S., et al.: Sigmoidal equation for lung and chest wall volume-pressure curves in acute respiratory failure. J. Appl. Physiol. 95(5), 2064-2071 (2003)

11 Schumann, S., Burcza, B., Haberthur, C., et al.: Estimating intratidal nonlinearity of respiratory system mechanics: a model study using the enhanced gliding-SLICE method. Physiol. Meas. 30(12), 1341-1356 (2009)

12 Kent, R.W., Woods, W.A., Salzar, R.S., et al.: The transient relationship between pressure and volume in the pediatric pulmonary system. J. Biomech. 42(11), 1656-1663 (2009)

13 Barbini, P., Cevenini, G., Avanzolni, G.: Nonlinear mechanisms determining expiratory flow limitation in mechanical 
ventilation: a model-based interpretation. Ann. Biomed. Eng. 31(8), 908-916 (2003)

14 Antonaglia, V., Lucangelo, U., Ristagno, G., et al.: Gas distribution in a two-compartment model during volume or pressure ventilation: role of elastic elements. Respir. Physiol. Neurobiol. 171(3), 225-231 (2010)

15 Nucci, G., Tessarin, S., Cobelli, C.: A morphometric model of lung mechanics for time-domain analysis of alveolar pressures during mechanical ventilation. Ann. Biomed. Eng. 30(4), 537-545 (2002)

16 Polak, A.G., Mroczka, J.: Nonlinear model for mechanical ventilation of human lungs. Comput. Biol. Med. 36(1), 41-58 (2006)

17 Lo, P., Sporring, J., Ashraf, H., et al.: Vessel-guided airway tree segmentation: A voxel classification approach. Med. Image Anal. 14(4), 527-538 (2010)

18 Comerford, A., Forster, C., Wall, W.A.: Structured tree impedance outflow boundary conditions for 3D lung simulations. J. Biomech. Eng. 132(8), 081002 (2010)

19 Wilson, A.J., Murphy, C.M., Brook, B.S., et al.: A computer model of the artificially ventilated human respiratory system in adult intensive care. Med. Eng. Phys. 31(9), 1118-1133 (2009)

20 Yin, Y., Choi, J., Hoffman, E.A., et al.: Simulation of pulmonary air flow with a subject-specific boundary condition. J. Biomech. 43(11), 2159-2163 (2010)

21 Harris, R.S.: Pressure-volume curves of the respiratory system. Respir. Care 50(1), 78-98 (2005)

22 Smith, B., Rees, S., Tvorup, J., et al.: Modeling the influence of the pulmonary pressure-volume curve on gas exchange. Conf. Proc. IEEE Eng. Med. Biol. Soc. 3(23), 57-60 (2005)

23 Schirrmann, K., Mertens, M., Kertzscher, U., et al.: Theoretical modeling of the interaction between alveoli during inflation and deflation in normal and diseased lungs. J. Biomech. 43(6), 1202-1207 (2010)

24 Fenn, W.O., Rahn, H., Otis, A.B.: A theoretical study of the composition of the alveolar air at altitude. Am. J. Physiol. 146(5), 637-653 (1946)

25 Aboab, J., Louis, B., Jonson, B., et al.: Relation between $\mathrm{PaO}_{2} / \mathrm{FIO}_{2}$ ratio and $\mathrm{FIO}_{2}$ : a mathematical description. Intensive Care Med. 32(10), 1494-1497 (2006)

26 Thomas, V., Costes, F., Busso, T.: Estimation of arterial $\mathrm{PCO}_{2}$ from a lung model during ramp exercise in healthy young subjects. Respir. Physiol. Neurobiol. 156(3), 259-265 (2007)

27 Benallal, H., Beck, K.C., Johnson, B.D., et al.: Evaluation of cardiac output from a tidally ventilated homogeneous lung model. Eur. J. Appl. Physiol. 95(2-3), 153-162 (2005)

28 Roughton, F.J., Forster, R.E.: Relative importance of diffusion and chemical reaction rates in determining rate of exchange of gases in the human lung, with special reference to true diffusing capacity of pulmonary membrane and volume of blood in the lung capillaries. J. Appl. Physiol. 11(2), 290-302 (1957)

29 Patel, S.: Evaluation of the resistance of membrane and erythrocytes to oxygen transport in pulmonary capillaries. Respir. Physiol. Neurobiol. 130(2), 181-187 (2002)

30 Steimle, K.L., Mogensen, M.L., Karbing, D.S., et al.: A model of ventilation of the healthy human lung. Comput. Methods Programs Biomed. (2010)

31 Krogh, A., Lindhard, J.: The volume of the dead space in breathing and the mixing of gases in the lungs of man. J. Physiol. 51(1-2), 59-90 (1917)

32 Wagner, P.D.: The multiple inert gas elimination technique (MIGET). Intensive Care Med. 34(6), 994-1001 (2008)

33 Anderson, J.C., Hlastala, M.P.: Impact of airway gas exchange on the multiple inert gas elimination technique: theory. Ann. Biomed. Eng. 38(3), 1017-1030 (2010)

34 Benallal, H., Busso, T.: Analysis of end-tidal and arterial $\mathrm{PCO}_{2}$ gradients using a breathing model. Eur. J. Appl. Physiol. 83(45), 402-408 (2000)

35 Whiteley, J.P., Gavaghan, D.J., Hahn, C.E.: Mathematical modeling of pulmonary gas transport. J. Math. Biol. 47(1), 79-99 (2003)

36 Ben-Tal, A.: Simplified models for gas exchange in the human lungs. J. Theor. Biol. 238(2), 474-495 (2006)

37 Rees, S.E., Kjaergaard, S., Andreassen, S., et al.: Reproduction of MIGET retention and excretion data using a simple mathematical model of gas exchange in lung damage caused by oleic acid infusion. J. Appl. Physiol. 101(3), 826-832 (2006)

38 Niklason, L., Eckerstrom, J., Jonson, B.: The influence of venous admixture on alveolar dead space and carbon dioxide exchange in acute respiratory distress syndrome: computer modelling. Crit. Care 12(2), R53 (2008)

39 Qiu, A., Bai, J.: Multiple modeling in the study of interaction of hemodynamics and gas exchange. Comput. Biol. Med. 31(1), 59-72 (2001)

40 Ben-Tal, A., Smith, J.C.: A model for control of breathing in mammals: coupling neural dynamics to peripheral gas exchange and transport. J. Theor. Biol. 251(3), 480-497 (2008)

41 Lu, K., Clark, J.W., Jr., Ghorbel, F.H., et al.: A human cardiopulmonary system model applied to the analysis of the Valsalva maneuver. Am. J. Physiol. Heart Circ. Physiol. 281(6), H2661-79 (2001)

42 Montebelli, G.: Effects of expiratory flow limitation on mechanical cardio-pulmonary interactions: a model study. Politecnico di Milano (2005)

43 Fontecave J.J., Abdulhay, E., Calabrese, P., et al.: A model of mechanical interactions between heart and lungs. Philos. Transact. A Math. Phys. Eng. Sci. 367(1908), 4741-4757 (2009)

44 Ben-Tal, A., Smith, J.C.: Control of breathing: two types of delays studied in an integrated model of the respiratory system. Respir. Physiol. Neurobiol. 170(1), 103-112 (2010) 